\title{
磺酰叠氮和炔参与的多组分反应研究进展
}

\author{
张文生 $*, a$ 许文静 ${ }^{a}$ 匡春香 $b$ \\ $\left({ }^{a}\right.$ 焦作师范高等专科学校理工学院 焦作 454001) \\ ( ${ }^{b}$ 同济大学化学系 上海 200092)
}

\begin{abstract}
摘要 磺酰叠氮和炔参与的铜催化多组分反应成为近年来有机化学研究的热点. 磺酰叠氮和炔在铜催化下生成的 $N$-磺 酰基烯酮亚胺中间体，可以被胺、醇和水等各类亲核试剂捕捉，也可以和各种类型的烯烃发生[2+2]、[3+2]和[4+2] 等环加成反应. $N$-磺酰基烯酮亚胺中间体受到来自双官能团底物的分子内基团进攻以及随后发生的(环)重排和 $\sigma$ 键迁 移, 更是成为构建结构丰富的具有生理和药物活性(杂)环类化合物的重要手段. 主要对该领域近年来的最新研究成果 进行了综述.
\end{abstract}

关键词 磺酰叠氮; 炔; 烯酮亚胺; 多组分反应; 铜催化

\section{Research Progress on Multicomponent Reactions of Sulfonyl Azides and Terminal Alkynes}

\author{
Zhang, Wensheng ${ }^{*, a} \quad \mathrm{Xu}$, Wenjing $^{a} \quad$ Kuang, Chunxiang ${ }^{b}$ \\ ( ${ }^{a}$ School of Technology, Jiaozuo Teachers College, Jiaozuo 454001) \\ ( ${ }^{b}$ Department of Chemistry, Tongji University, Shanghai 200092)
}

\begin{abstract}
In recent years, the copper-catalyzed multicomponent reactions involving sulfonyl azides and terminal alkynes have received much attention in organic chemistry. $\mathrm{N}$-Sulfonyl ketenimine intermediates, generated in situ from the copper(I)-catalyzed cycloaddition of sulfonyl azides and terminal alkynes, could be trapped by various nucleophiles such as amines, alcohols and $\mathrm{H}_{2} \mathrm{O}$, etc. In addition, the ketenimine intermediates could also react with a wide range of olefins though $[2+2],[3+2]$ and [4+2] cycloadditions. Moreover, attack of the ketenimine by intramolecular nucleophilic functional group and the following rearrangement or $\sigma$-shift course have become a powerful tool for the construction of diverse biologically and pharmacologically active heterocyclic compounds. In this paper, the research achievements recently in the important area are described.
\end{abstract}

Keywords sulfonyl azide; alkyne; ketenimine; multicomponent reaction; copper-catalyzed

多组分反应具有原子经济性、环境友好、操作简便、 反应效率高等优点, 近年来越来越引起化学家们的重视 和关注 ${ }^{[14]}$. 磺酰叠氮和炔参与的多组分反应近年来成 为铜催化有机反应的研究热点. Chang 和王彦广课题组 等对此类反应做了大量的研究. 他们发现磺酰叠氮等含 有吸电子基团的有机叠氮化合物和炔发生铜催化环加 成(CuACC)反应时, 生成五元环中间体不稳定. 该中间 体释放出 1 分子的 $\mathrm{N}_{2}$ 而得到一种有价值的含氮累积二
烯烃中间体一一 $N$-磺酰基烯酮亚胺. $N$-磺酰基烯酮亚胺 中间体可以被胺 ${ }^{[5]}$ 、水 ${ }^{[6-8]}$ 和醇 ${ }^{[9,10]}$ 等亲核试剂捕捉, 在 室温下完成多组分反应(Scheme 1). $N$-磺酰基烯酮亚胺 中间体具有足够的半衰期和相对稳定性, 还可以参与环 加成反应、分子内电环化或重排反应，构建包括四元环 在内的更多有价值的杂环化合物. Chang 和王彦广等分 别对 2012 年以前有关烯酮亚胺中间体的生成和合成应 用进行了综述 ${ }^{[11}$ 14]. 本文主要对 2012 年以后的最新研

\footnotetext{
* E-mail: tongjizws@163.com

Received May 6, 2015; revised June 2, 2015; published online June 16, 2015.

Project supported by the National Natural Science Foundation of China (No. 21272174), the Foundation of He'nan Scientific and Technological Committee (No. 142300410338), the Science and Technology Research Key Program of Henan Educational Committee (No. 14B150056) and the Foundation of Jiaozuo Scientific and Technological Bureau (No. 2014400038).

国家自然科学基金(No. 21272174)、河南省自然科学基金(No. 142300410338)、河南省教育厅自然科学重点研究计划(No. 14B150056)和焦作市科学技 术局科技计划(No. 2014400038)资助项目.
} 
究成果进行综述和展望.

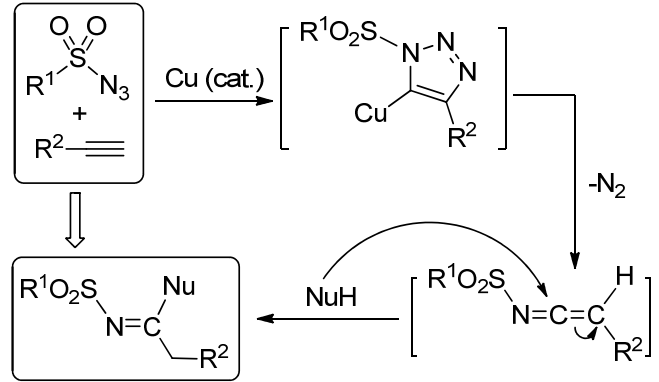

图式 1 磺酰叠氮和炔参与的多组分反应

Scheme 1 Multicomponent reactions involving sulfonyl azides and terminal alkynes

\section{1 磺酰叠氮和炔参与的三组分反应}

\section{1 分子间三组分反应}

1.1.1 第三组分和 $N$-磺酰基烯酮亚胺的环加成

马成课题组 ${ }^{[15]}$ 以磺酰叠氮、炔和炔醛为原料进行三 组分反应，发现氢氧化锂可使 $N$-磺酰基烯酮亚胺中间 体转化为 $N$-磺酰基炔氨基锂中间体. 该中间体再和炔 醛发生 $[2+2]$ 环加成和酸作用下的四元环的开环, 可以 合成炔烯共轭结构的 $N$-磺酰基酰胺(Scheme 2).<smiles>[R]C#C[R]#CC(=O)C#[R]</smiles>

(1) Cul (cat.)

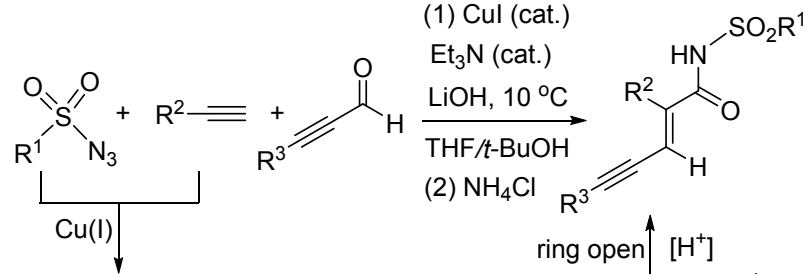

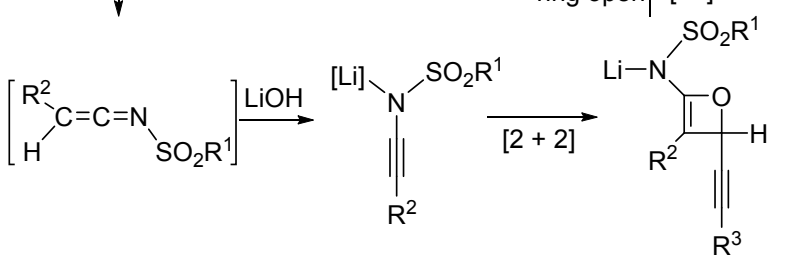

图式 2 磺酰叠氮、炔和炔醛的三组分反应

Scheme 2 Three-component reactions of sulfonyl azides, terminal alkynes and ynals

Yavari 等 ${ }^{[16]}$ 以磺酰叠氮、炔和酰氯的腙为原料进行 铜催化三组分反应, 酰氯的腙和 $N$-磺酰基烯酮亚胺中 间体的烯键发生 $[3+2]$ 环加成反应, 得到了四取代的吡 唑类化合物(Scheme 3).

王彦广等 ${ }^{[17]}$ 以亚胺酰氯、磺酰叠氮和 2 equiv. 的炔 为原料进行三组分反应. 炔在铜催化下分别和前两种底

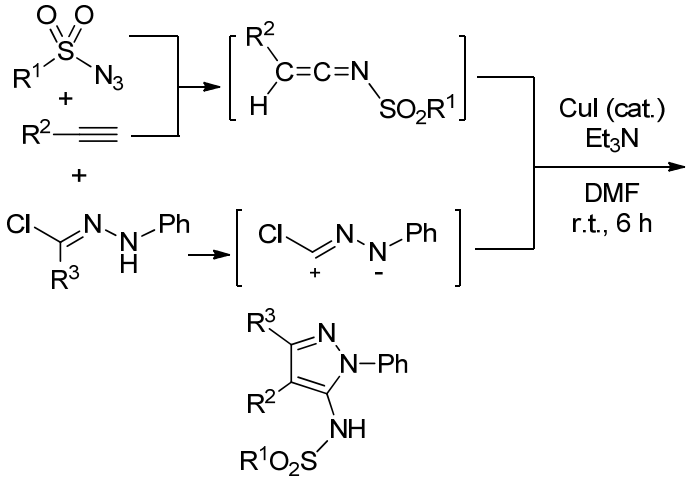

图式 3 磺酰叠氮、炔和酰氯的腙的三组分反应

Scheme 3 Three-component reactions of sulfonyl azides, terminal alkynes and hydrazonoyl chlorides

物生成烯酮亚胺和炔基亚胺两种中间体，两种中间体之 间的 $[2+2]$ 环加成反应得到的是多取代氮杂环丁烷类产 物(Scheme 4).

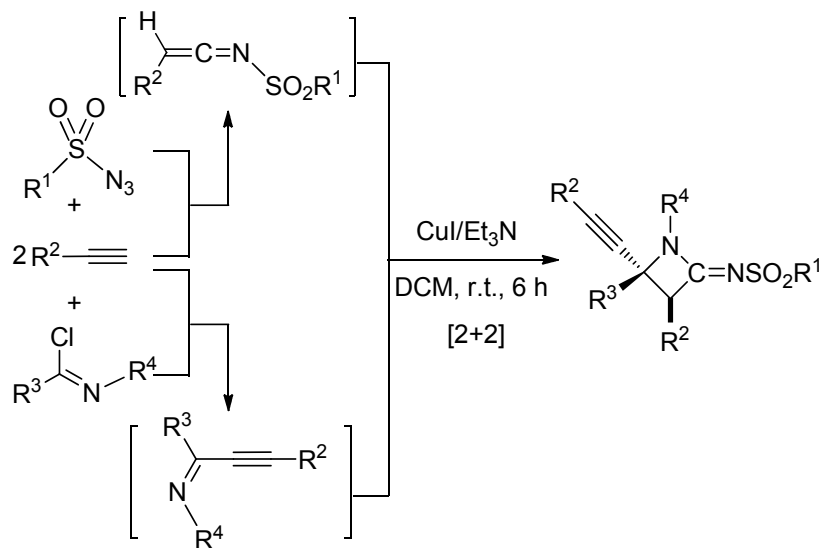

图式 4 磺酰叠氮、炔和亚胺酰氯的三组分反应

Scheme 4 Three-component reactions of sulfonyl azides, terminal alkynes and imidoyl chlorides

Pitchumani 等 ${ }^{[18]}$ 以 $N$-芳基亚甲基吡啶-2-胺为原料, 和磺酰叠氮、炔进行铜催化的三组分反应，通过 $[4+2]$ 环加成和氧化脱氢过程，构建了吡啶 $[1,2-a]$ 嘧啶-4-亚胺 类化合物(Scheme 5).

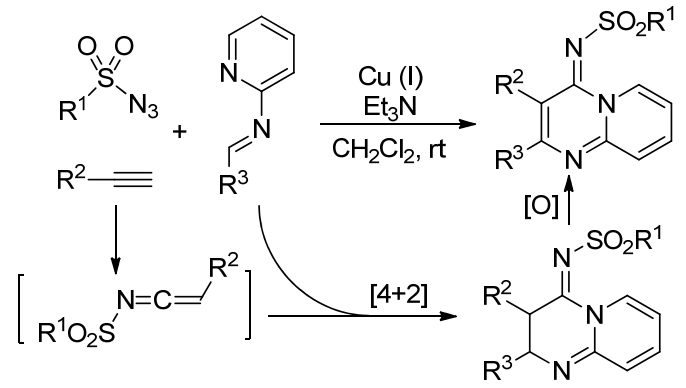

图式 5 磺酰叠氮、炔和 $N$-芳基亚甲基吡啶-2-胺的三组分反 应

Scheme 5 Three-component reactions of sulfonyl azides, terminal alkynes and $N$-arylidenepyridin-2-amines 
Shanmugam 等 ${ }^{[19]}$ 以二茂铁甲醛(酮)的 Schiff 碱和磺 酰叠氮、炔为原料, 通过发生在烯酮亚胺中间体的烯键 和 Schiff 碱结构中的 $\mathrm{C}=\mathrm{N}$ 键之间的[4+2]环加成合成 了含二茂铁的氮杂环丁烷-2-亚胺类化合物(Scheme 6).

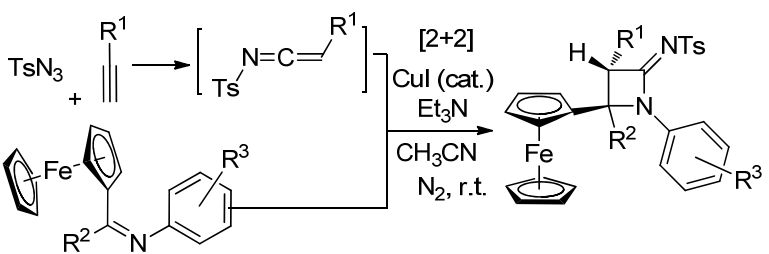

图式 6 磺酰叠氮、炔和二茂铁甲醛(酮)的 Schiff 碱的三组分 反应

Scheme 6 Three-component reactions of sulfonyl azides, terminal alkynes and ferrocenylimines

商永嘉课题组 ${ }^{[20]}$ 以 2-氨基硫酚的 Schiff 碱和磺酰叠 氮、炔为原料, 通过 $N$-磺酰基烯酮亚胺的烯键和 Schiff 碱的碳氮双键的 $[2+2]$ 环加成, 合成了含二硫键的氮杂 环丁烷-2-亚胺类化合物. 三组分反应的 CuI 体系促进了 颈基的二聚过程, 从而得到的是一个通过二硫键连接的 双含氮四元环结构(Scheme 7).
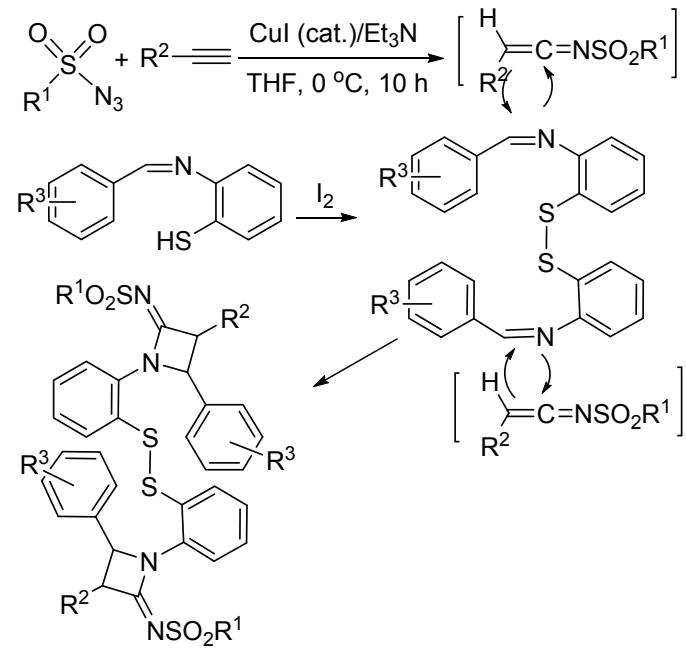

图式 7 磺酰叠氮、炔和 2-氨基硫酚的 Schiff 碱的三组分反应 Scheme 7 Three-component reactions of sulfonyl azides, terminal alkynes and 2-aminothiophenol derived Schiff base

Yavari 等 ${ }^{[21]}$ 以磺酰叠氮、炔和偶氮二甲酸酯为原料, 通过偶氮二甲酸酯的 $\mathrm{N}=\mathrm{N}$ 键和 $N$-磺酰基烯酮亚胺中 间体烯键的 $[2+2]$ 环加成反应和四元环的异构化开环闭环过程, 构建的是一种 4-磺酰亚胺基咪唑-2-酮类化 合物(Scheme 8).

Shanmugam 等 ${ }^{[22]}$ 利用 $N$-甲基吲哚醌-2-亚胺、磺酰 叠氮和炔的三组分反应, 通过亚胺的 $\mathrm{C}=\mathrm{N}$ 键和 $N$-磺酰 基烯酮亚胺中间体烯键的 $[2+2]$ 环加成, 构建了氮杂环 丁烷和吲哚 2-酮螺接而成的螺环化合物(Scheme 9).

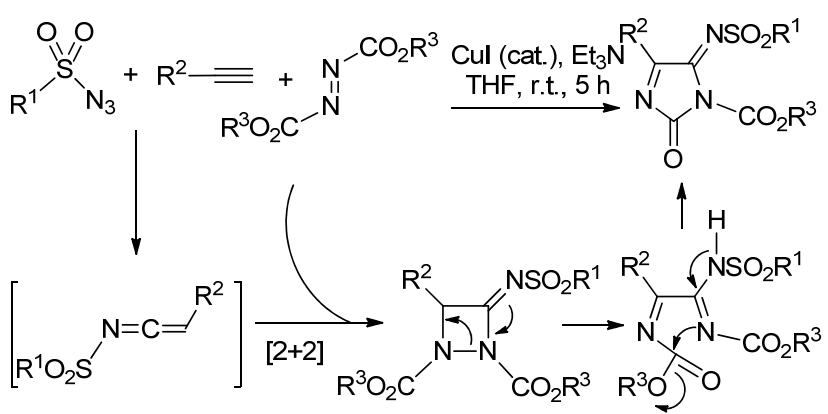

图式 8 磺酰叠氮、炔和偶氮二甲酸酯的三组分反应

Scheme 8 Three-component reactions of sulfonyl azides, terminal alkynes and dialkyl azodicarboxylates

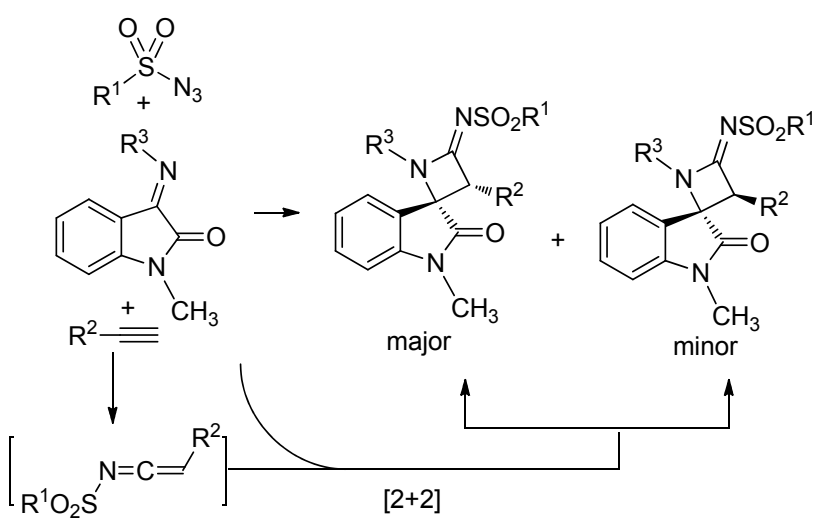

图式 9 磺酰叠氮、炔和 $N$-甲基吲哚醌-2-亚胺的三组分反应 Scheme 9 Three-component reactions of sulfonyl azide s, terminal alkynes and $N$-methylisatinimines

\subsection{2 第三反应组分捕捉 $N$-磺酰基烯酮亚胺中间体}

张玉红课题组 ${ }^{[23]}$ 报道了 $N, N$-二甲基-二甲氧基甲胺 和对甲基苯磺酰叠氮、芳炔之间的三组分反应. $N, N$-二 甲基-二甲氧基甲胺首先和炔作用，在消去 $\mathrm{MeOH}$ 的同 时得到一个亚铵中间体. 与传统的亲核试剂捕捉 $N$-磺 酰基烯酮亚胺中间体的中心碳原子不同，该中间体则是 正电进攻中间体的端基碳原子，再经历 $\mathrm{N}$ 原子的分子内 进攻中心碳原子和重排等复杂过程得到 $N$-磺酰基- $\alpha, \beta$ 不饱和的榺类化合物(Scheme 10).

最近，Yavari 等 ${ }^{[24]}$ 通过 2-氨基苯并咪唑的氨基来亲 核捕捉由磺酰叠氮和炔产生的 $N$-磺酰基烯酮亚胺中间 体, 获得 2-苯并咪唑- $N$-磺酰基脒类化合物(Scheme 11).

\section{2 分子间两组分反应-分子内串联反应}

近年来, 化学家们更多的关注磺酰叠氮和含有双官 能团的芳炔两种底物之间反应研究. 芳炔邻位的亲核基 团对磺酰叠氮和炔形成的 $N$-磺酰基烯酮亚胺中间体进 行分子内的亲核进攻，通过闭环合成了有价值的杂环化 合物.

吴款教授等 ${ }^{[25]}$ 报道了 2 -乙炔芳基氮丙啶和环氧乙 烷衍生物分别与磺酰叠氮的分子间两组分反应-分子内 


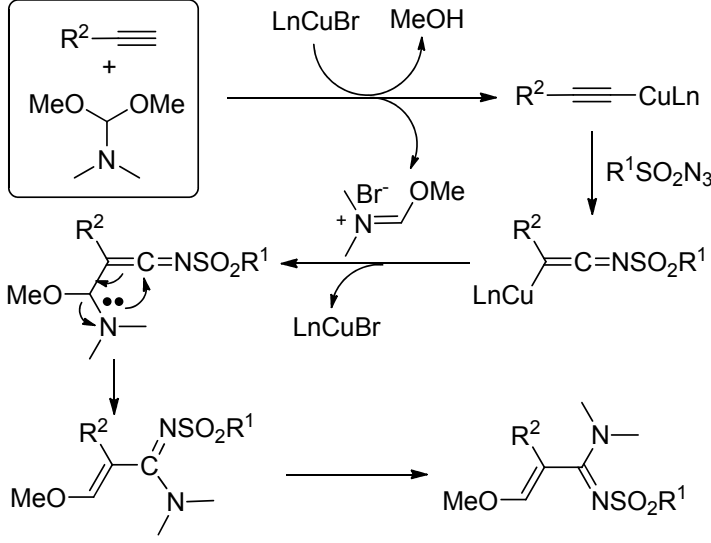

图式 10 磺酰叠氮、炔和 $N, N$-二甲基-二甲氧基甲胺的三组分 反应

Scheme 10 Three-component reactions of sulfonyl azides, terminal alkynes and $N, N$-dialkyloxyformamidedialkyl acetals

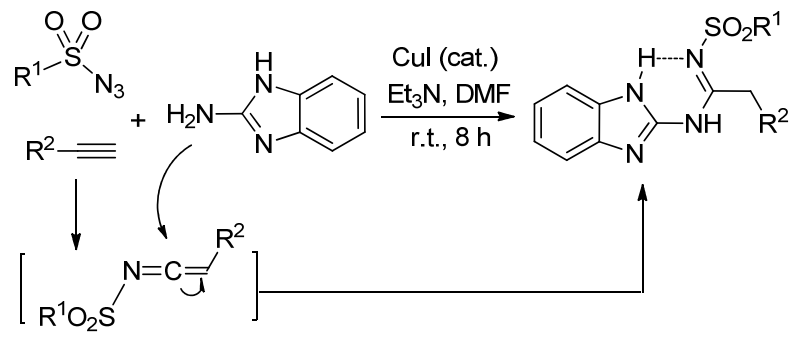

图式 11 磺酰叠氮、炔和 2-氨基苯并咪唑的三组分反应

Scheme 11 Three-component reactions of sulfonyl azides, terminal alkynes and 2-amino-benzoimidazoles

串联反应. 三元环中的 $\mathrm{N} / \mathrm{O}$ 原子分子内亲核进攻 $N$-磺 酰基烯酮亚胺中间体闭合首先形成一个和三元环桥联 的七元环, 然后再发生三元环开环, 分别构建了七元杂 环苯并氮杂环庚二烯或苯并氧杂环庚二烯(Scheme 12).

涂永强和曹小平等 ${ }^{[26]}$ 通过铜催化的分子内 1,6 -烯 炔的炔键和磺酰叠氮首先生成烯酮亚胺中间体, 然后在 两个烯键之间发生 $[2+2]$ 环加成反应，构建了一类二环 [3.1.1]庚烷-6-酮结构的化合物(Scheme 13).

崔秀灵等 ${ }^{[27]}$ 以炔丙基亚胺为原料和磺酰叠氮反应, 通过生成 $N$-磺酰基烯酮亚胺中间体的 $6 \pi$-电环化和 [1,3]-H-迁移的串联反应，构建了 4-磺酰胺基喹啉结构 化合物(Scheme 14).

王彦广等 ${ }^{[28]}$ 以 $N$-炔丙基酰胺和磺酰叠氮为原料, $\mathrm{MeCN}$ 为反应溶剂, $4 \AA \mathrm{MS}$ 为脱水剂, 在 CuI-pyridine 体系催化和 $\mathrm{N}_{2}$ 保护下 $70{ }^{\circ} \mathrm{C}$ 反应 $1 \sim 3 \mathrm{~h}$, 得到了 3-磺酰 基嘧啶-4-酮类化合物(Scheme 15). 该反应通过酰胺键 中的羰基氧原子分子内进攻炔和磺酰叠氮形成的烯酮 亚胺中间体闭合成六元环, 然后再经历一次 $\mathrm{C}-\mathrm{O}$ 键断 裂的开环和 $\mathrm{C}-\mathrm{N}$ 键形成的关环过程. Lin 等 ${ }^{[29]}$ 发现

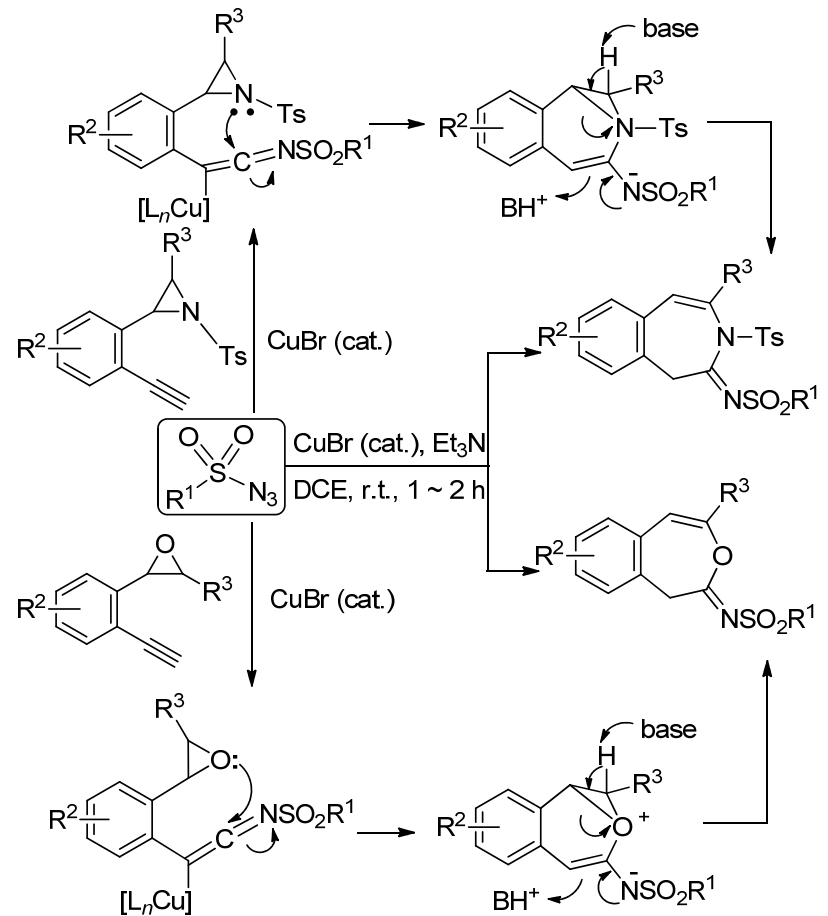

图式 12 铜催化的磺酰叠氮与 2-乙炔芳基氮丙啶或 2-乙炔芳 基环氧乙烷反应

Scheme 12 Cu-catalyzed reactions of 2-(2-ethynylphenyl)-1tosylaziridine or 2-(2-ethynylphenyl)oxirane with sulfonyl azides

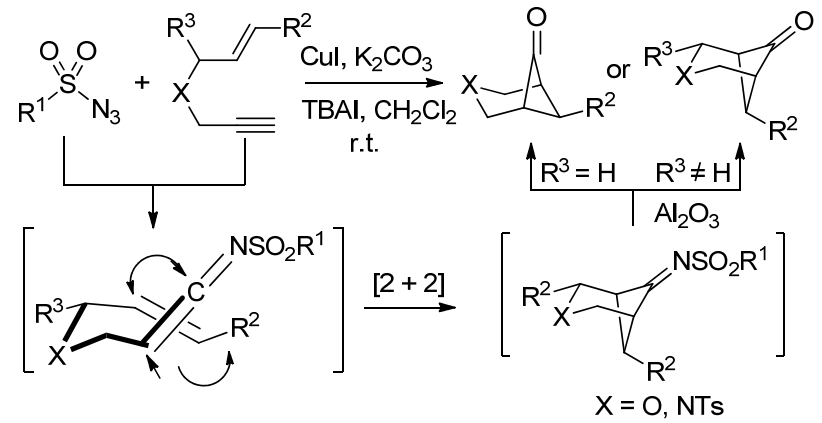

图式 13 铜催化的 1,6-烯炔烯酮亚胺分子内 $[2+2]$ 环加成反 应

Scheme 13 Cu-catalyzed intramolecular [2+2] cycloaddition of 1,6-enyne-derived ketenimine

以 $\mathrm{CH}_{2} \mathrm{Cl}_{2}$ 为反应溶剂，同样的反应底物在 $\mathrm{CuI}-\mathrm{K}_{2} \mathrm{CO}_{3}$ 体系催化下室温反应 $12 \mathrm{~h}$, 可得到同样产物. 他们使用 树脂吸附反应后的铜催化剂和直接过滤的方法得到粗 产品，经洗涤后可以更高收率的得到纯净的目标产物.

王彦广等 ${ }^{\left[{ }^{[0]}\right.}$ 以邻乙炔基苯甲醛的缩醛和磺酰叠氮 为原料，通过 $N$-磺酰基烯酮亚胺中间体合成 1,2-二氢异 喹啉结构的化合物. 环状缩醛底物的 $N$-磺酰基烯酮亚 胺中间体发生 1,5- $H$ 迁移后闭合环化, 而非环状缩醛底 物的烯酮亚胺中间体则发生 1,5-OR 迁移后闭合环化, 因而分别得到的是 1,1-和 1,3-二烷氧基的两种产物 


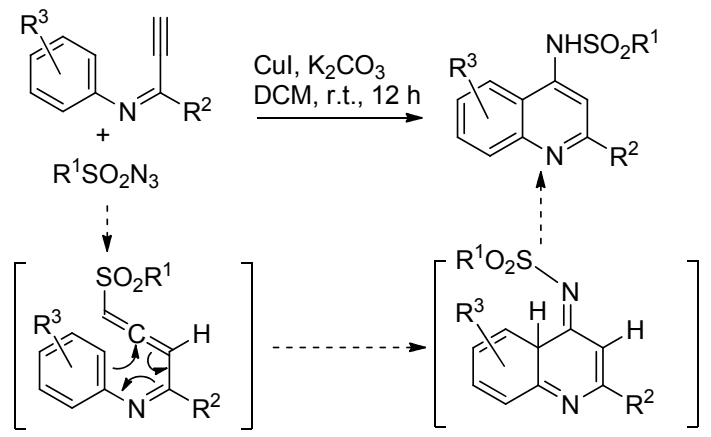

图式 14 铜催化的磺酰叠氮炔和丙基亚胺反应

Scheme 14 Cu-catalyzed reaction of sulfonyl azides with alkynyl imines

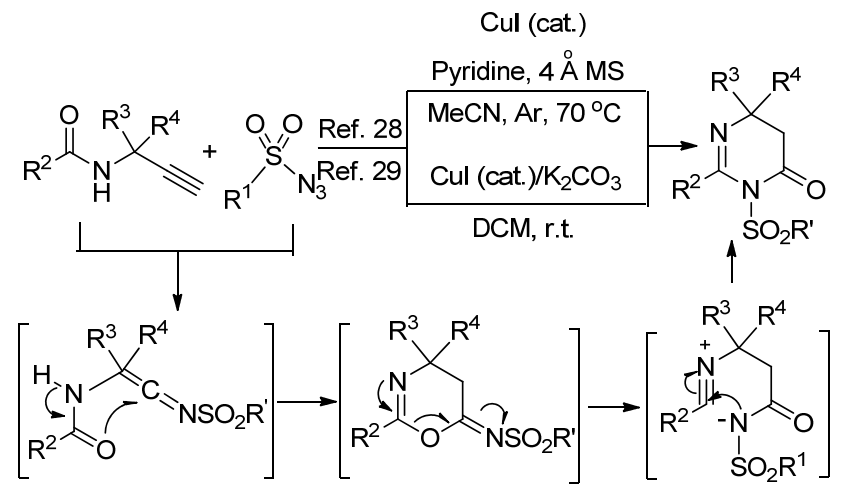

图式 15 铜催化的磺酰叠氮炔和 $N$-炔丙基酰胺反应

Scheme 15 Cu-catalyzed reaction of sulfonyl azides with $N$-(prop-2-yn-1-yl)amides

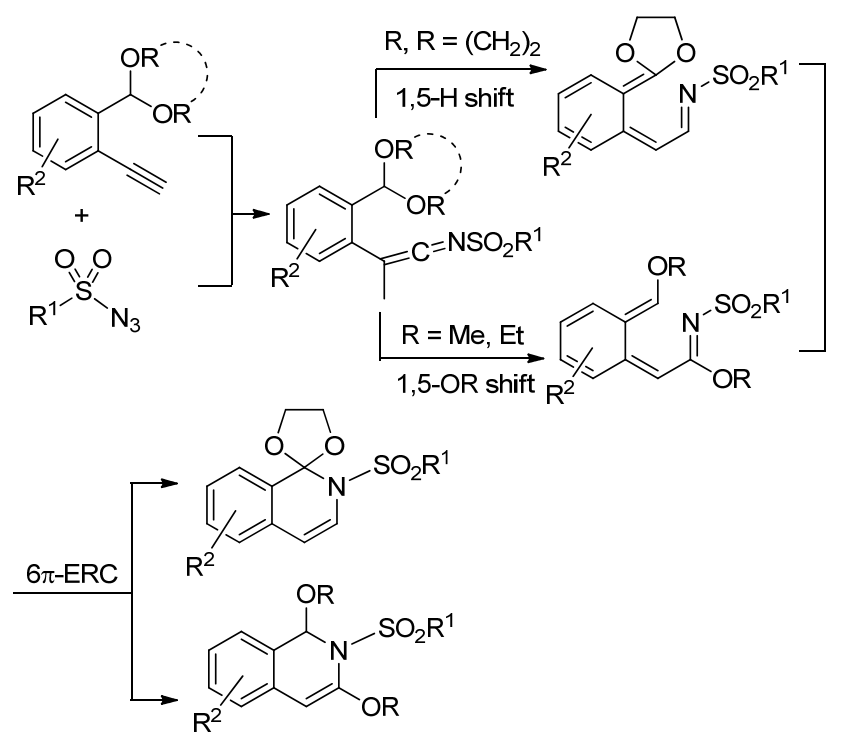

图式 16 铜催化的磺酰叠氮炔和邻乙炔基苯甲缩醛反应 Scheme 16 Cu-catalyzed reaction of sulfonyl azides with $o$-ethynylbenzacetals

(Scheme 16).

Reddy 等 $^{[31]}$ 研究了炔丙醇乙酸酯和磺酰叠氮之间 的反应, 得到了 $\alpha, \beta$-不饱和酰胺化合物(Scheme 17). 他
们认为炔丙醇乙酸酯的端基炔键和磺酰叠氮生成的 $N$ 磺酰基烯酮亚胺中间体，受到酯键羰基氧原子的分子内 亲核进攻, 发生 [3,3]- $\sigma$ 键迁移(1,3-乙酰基迁移)和酯的 水解, 得到了目标化合物.

$$
\text { MeCN, air }
$$

图式 17 铜催化的磺酰叠氮炔和炔丙醇乙酸酯反应 Scheme $17 \mathrm{Cu}$-catalyzed reaction of sulfonyl azides with propargyl acetates

Nakamura 等 ${ }^{[32]}$ 研究了 $O$-炔丙基肜底物和磺酰叠氮 的反应. 磺酰叠氮和炔键先形成 $N$-磺酰基烯酮亚胺中 间体，再接受分子内肟的 $\mathrm{N}$ 原子进攻. 整个反应历程经 历了闭合为五元环、开环、再次闭合为六元环和再次开 环的一系列复杂过程, 最后得到稳定的 $\alpha, \beta$-不饱和的脒 类结构的产物(Scheme 18).

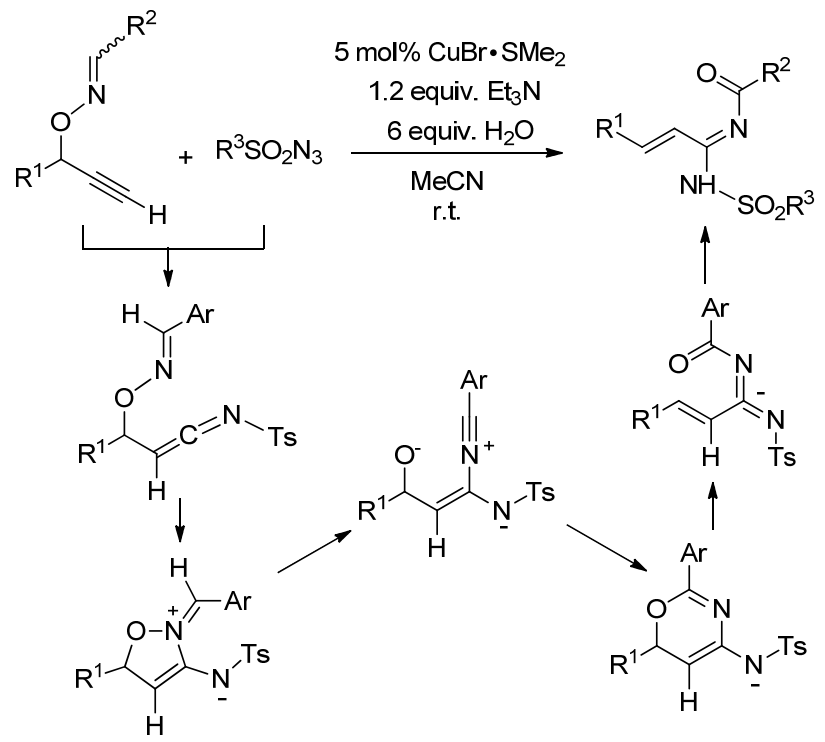

图式 18 催化的磺酰叠氮炔和 $O$-炔丙基肜底物反应 Scheme 18 Cu-catalyzed reaction of sulfonyl azides with $O$-propargylic oximes

\section{2 磺酰叠氮和炔参与的四组分反应}

\section{1 分子间四组分反应}

Shanmugam 等 ${ }^{[33]}$ 通过对甲基苯磺酰叠氮、芳炔、 苯肼和醛之间的四组分反应. 四组分中的苯肼和醛先缩 合为腙, 腙的饱和氮原子亲核进攻由磺酰叠氮和芳炔生 
成的烯酮亚胺中间体, 合成了腙取代的磺酰亚胺类化合 物(Scheme 19).

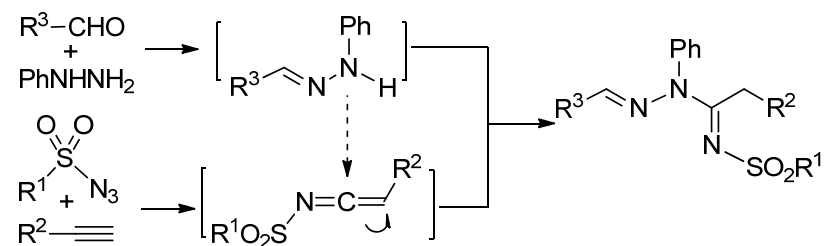

图式 19 对甲基苯磺酰叠氮、芳炔、苯肼和醛的四组分反应

Scheme 19 Four-component coupling reaction of aldehydes, alkynes, tosyl azides, and phenylhydrazines

\section{2 分子间三组分反应-分子内串联反应}

磺酰叠氮和芳炔参与的三组分, 还可以继续接受反 应底物中另一官能团的分子内进攻, 通过分子间两组分 反应-分子内串联反应，构建多种结构丰富的(杂)环化 合物。

在对 2-乙炔芳基环氧乙烷和磺酰叠氮发生分子间 两组分反应-分子内串联反应的研究基础上 ${ }^{[25]}$, 吴劼教 授等 ${ }^{[34]}$ 向反应体系中加入异腈化合物进行了多组分反 应. 该反应涉及到 $N$-磺酰基烯酮亚胺中间体的形成、环 氧乙烷氧原子的亲核进攻、三元环的开环重排为七元环 以及一个由异腈化合物参与进来的 $[3+2]$ 环加成等串联 过程, 构建了一类含 $\mathrm{N} 、 \mathrm{O}$ 的螺[4.6]杂环化合物(Scheme 20).<smiles>C#Cc1ccccc1C1(O)OC1P</smiles>

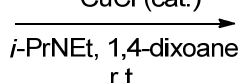
r.t.<smiles>[Y]NS(N)(=O)=O</smiles>

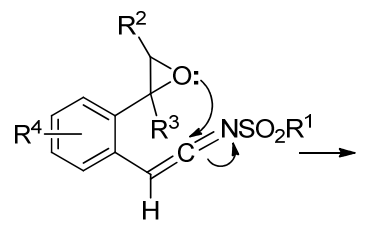<smiles>[R][Te]CC</smiles><smiles>[R12]O[N+](=O)[O-]</smiles>

图式 20 铜催化的磺酰叠氮、2-乙炔芳基环氧乙烷和异腈化 合物反应

Scheme 20 Cu-catalyzed reaction of 2-(2-ethynylphenyl)oxiranes, sulfonyl azides, with 2-isocyanoacetates

王彦广等 ${ }^{[35]}$ 选择邻碘代苯胺和磺酰叠氮、芳炔之间 进行三种底物的分子内四组分反应，将 $N$-磺酰基椫的 形成和 Pd-催化插羰偶联以及 Lewis 酸催化磺酰胺氢化 过程串联结合, “一锅法” 合成了 4(3H)-喹唑酮类化合 物(Scheme 21).
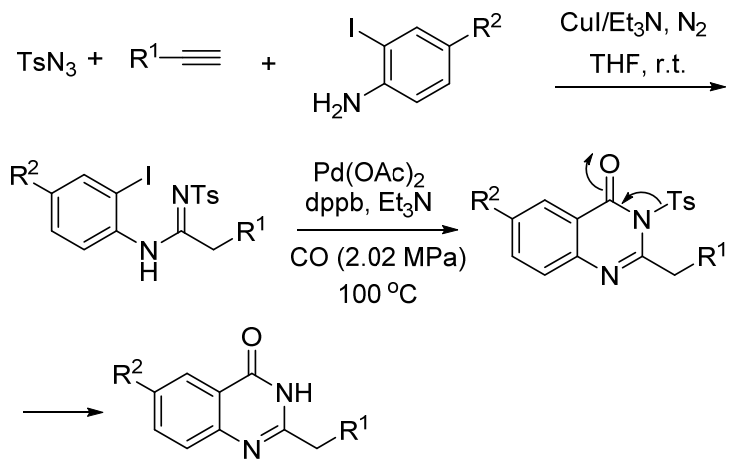

图式 21 铜催化的磺酰叠氮、芳炔和邻碘代苯胺反应 Scheme 21 Cu-catalyzed reaction of sulfonyl azides, alkynes and $o$-iodoanilines

王彦广等 ${ }^{[36]}$ 用炔丙基胺作为双官能团底物和磺酰 叠氮、炔发生分子内的四组分反应. 首先是氨基的 $\mathrm{N}$ 原 子捕捉磺酰叠氮和炔生成的 $N$-磺酰基烯酮亚胺中间体, 然后在碱的作用下炔键转化为累积二烯中间体，再通过 $6 \pi$-电环化和磺酰基的 1,3-转移得到 1,2,4,5-四取代的咪 唑衍生物(Scheme 22).

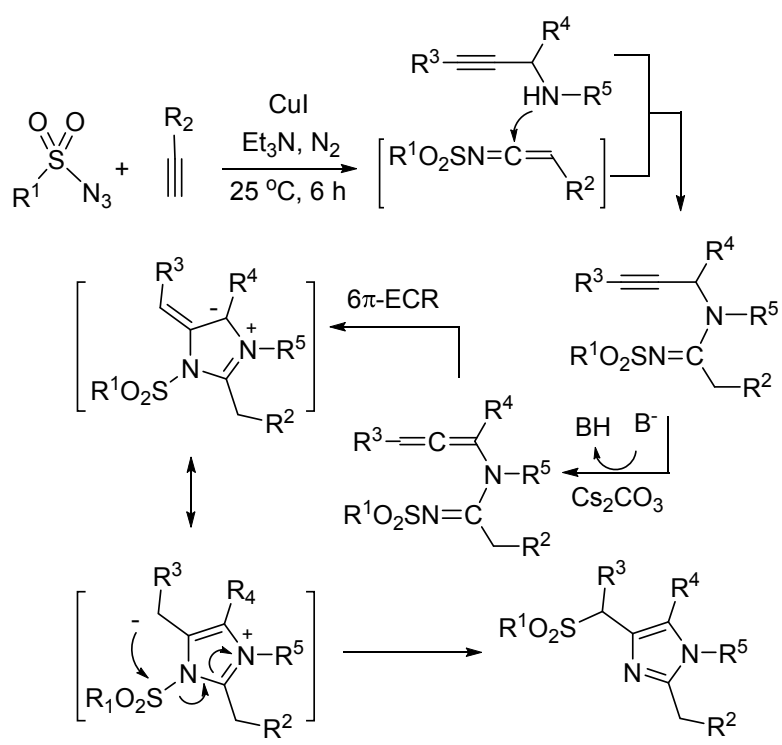

图式 22 铜催化的磺酰叠氮、芳炔和炔丙基胺反应

Scheme 22 Cu-catalyzed reaction of sulfonyl azides, alkynes and propargyl amines

Punniyamurthy 等 ${ }^{[37]}$ 以 2-炔丙氧基苯甲醛作为特殊 结构的炔类底物，和磺酰叠氮及酚之间进行分子内的四 组分反应。该反应的烯酮亚胺中间体没有直接受到 $\mathrm{ArOH}$ 的分子间亲核进攻，而是被分子内的醚键氧原子 首先捕捉，通过四元环中间体发生开环，接着受到酚氧 负离子进攻发生 1,4-加成，再和邻位的醛基发生羟醛缩 合反应，经过一系列的串联过程构建了苯并呋喃的环结 构(Scheme 23). 


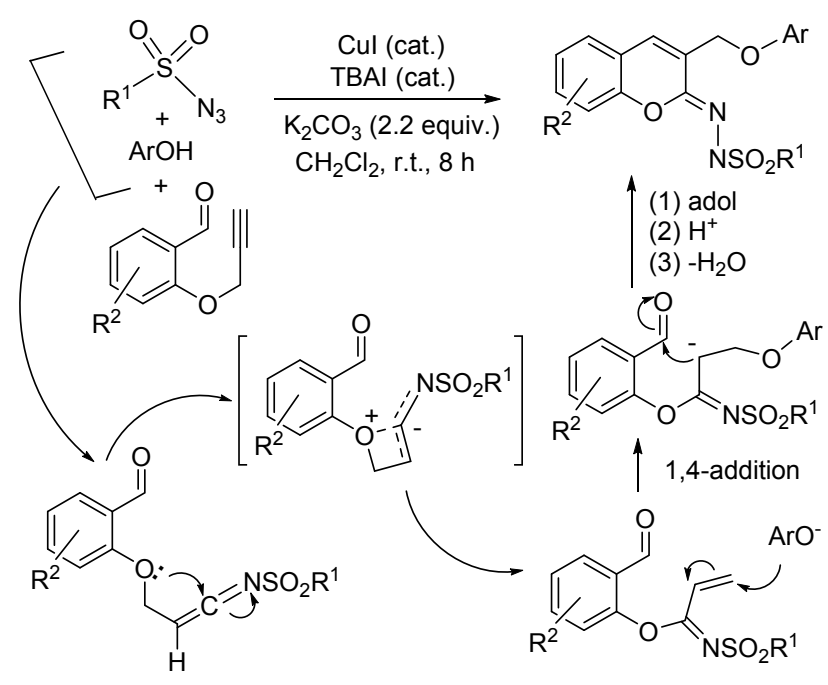

图式 23 铜催化的磺酰叠氮、芳炔和 2-炔丙氧基苯甲醛反应 Scheme 23 Cu-catalyzed reaction of sulfonyl azides, alkynes and ynals

张宁研究员等 ${ }^{[38]}$ 用 2-氨基亚甲基丙二腈类化合物 提供的氨基和腈基两个官能团，参与了和对甲基苯磺酰 叠氮、芳炔之间的分子内四组分反应. 该反应在室温和 $50{ }^{\circ} \mathrm{C}$ 分别下得到 4-氨基和 6-氨基取得的吡啶-2-亚胺类 化合物(Scheme 24). 他们提出反应的历程涉及烯酮亚 胺中间体受到丙二腈底物中的氨基进攻形成新的碳负 离子中间体, 后者再分子内进攻腈基环化为六元吡啶杂 环.

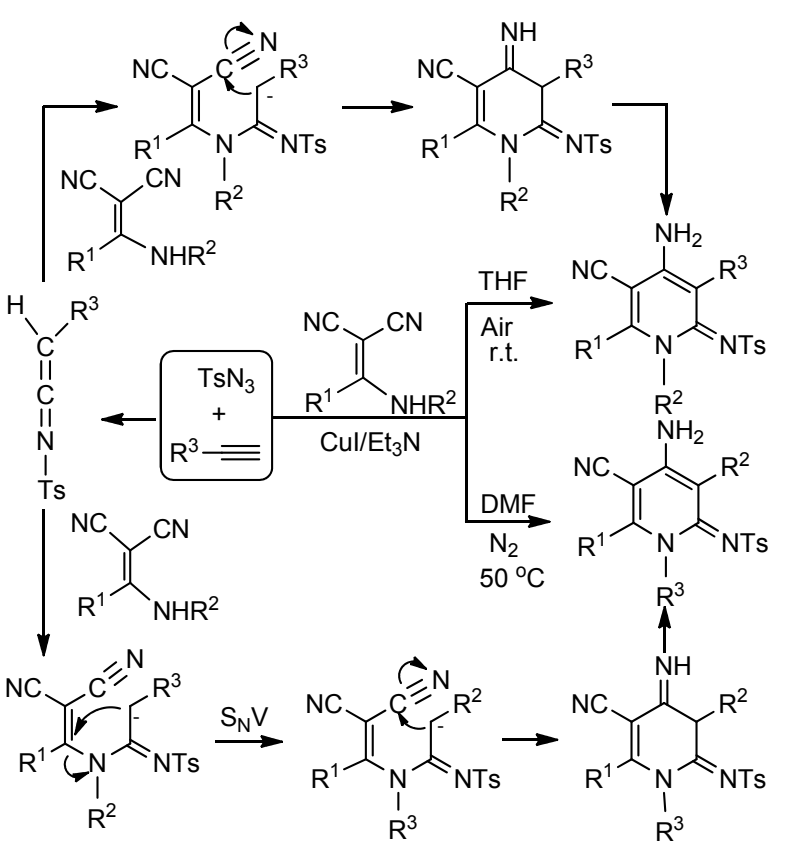

图式 24 铜催化的磺酰叠氮、芳炔和 2-氨基亚甲基丙二腈反 应

Scheme 24 Cu-catalyzed reaction of sulfonyl azides, alkynes and 2-[(amino)methylene]malononitriles
姜波和屠树江等 ${ }^{[39]}$ 研究了一个复杂结构的 1-(2-O吲哚-3-亚甲基)-2-芳氧基乙硫醇的三乙胺盐参与的多组 分反应. 该反应通过硫负离子进攻烯酮亚胺中心碳原子 得到的碳负离子中间体再亲核进攻芳基的 $\alpha$-位羰基闭 环，构建了一个新的功能化噻吩结构(Scheme 25).

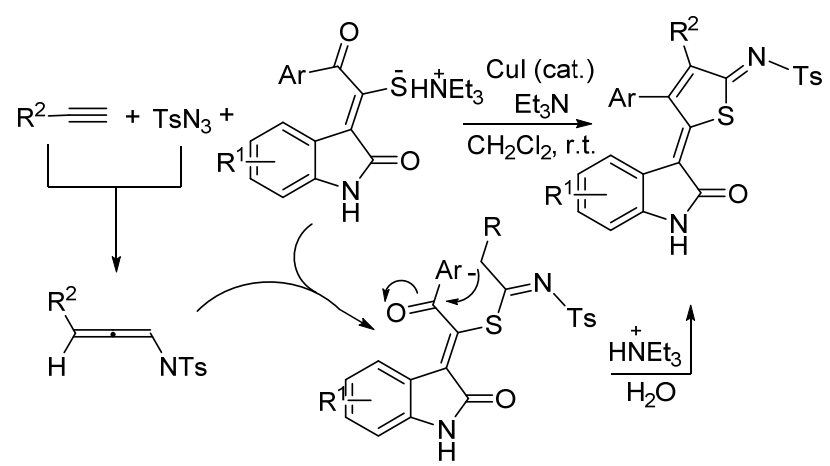

图式 25 铜催化的磺酰叠氮、芳炔和 1-(2- $O$-吲哚-3-亚甲 基)-2-芳氧基乙硫醇的三乙胺盐反应

Scheme 25 Cu-catalyzed reaction of sulfonyl azides, alkynes and triethylammonium 1-(2-oxoindolin-3-ylidene)-2-roylethaneathiolates

在王彦广等 ${ }^{[40]}$ 的研究基础上, Mandal 等 ${ }^{[41]}$ 用含糖 基的炔和磺酰叠氮、邻羟基苯甲醛反应，合成了一类含 糖的磺酰亚胺苯并吡喃(香豆素)型化合物(Scheme 26).

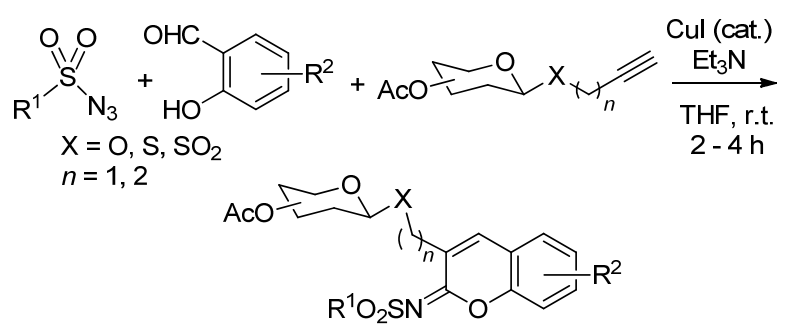

图式 26 铜催化的磺酰叠氮、含糖基的炔和邻羟基苯甲醛反 应

Scheme 26 Cu-catalyzed reaction of sulfonyl azides, sugar alkynes and salicylaldehydes

Pitchumani 等 ${ }^{[42]}$ 以磺酰叠氮、炔和 2-芳胺基丁烯二 酸为原料，通过芳胺基丁烯二酸的氨基进攻 $N$-磺酰基 烯酮亚胺中间体和随后的碳负离子分子内亲核取代反 应闭环和 $[1,3]-\mathrm{H}$ 迁移串联反应构建了功能化二氢吡咯 烷衍生物(Scheme 27).

\section{3 回顾与展望}

磺酰叠氮和炔参与的多组分反应的本质是首先通 过铜催化的 $[3+2]$ 环加成得到五元的三氮唑中间体发生 开环，得到一类活性的 $N$-磺酰基烯酮亚胺中间体. 由于 该过程释放出 1 分子的 $\mathrm{N}_{2}$, 具有不可逆性, 因此该类反 应大多数反应条件温和(一般为室温反应), 且反应效率 


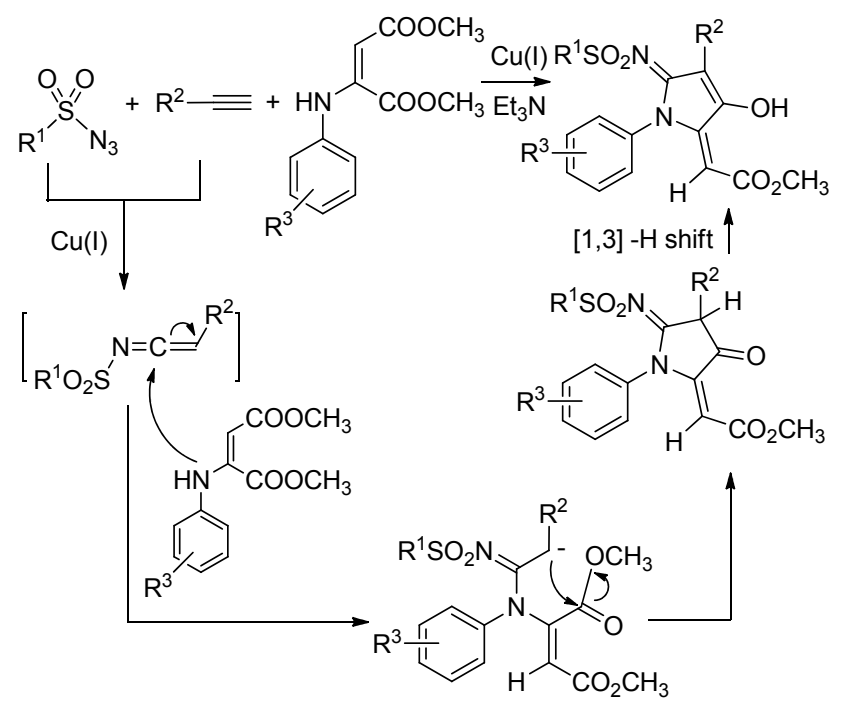

图式 27 铜催化的磺酰叠氮、炔和 2-芳胺基丁烯二酸反应

Scheme $27 \mathrm{Cu}$-catalyzed reaction of sulfonyl azides, alkynes and dimethyl 2-(phenylamino)maleates

较高. 此外, 使用低成本的铜催化多种组分一锅或串联 反应，极大地简化了反应和后处理程序，也是该类反应 的重要特征. 近年来, 该类反应的研究更趋向于分子内 的多组分反应, 在构建功能化的或具有潜在生理或药物 活性的杂环和多环化合物 ${ }^{[40]}$ 将发挥更加重要的作用.

\section{References}

[1] Estévez, V.; Villacampa, M.; Menéndez, J. C. Chem. Soc. Rev. 2010, 39, 4402.

[2] Touré, B. B.; Hall, D. G. Chem. Rev. 2009, 109, 4439.

[3] Cao, S.; Jing, Y.-F.; Liu, Y.-Y.; Wan J.-P. Chin. J. Org. Chem. 2014, 34, 876 (in Chinese). (曹硕, 景艳锋, 刘云云, 万结平, 有机化学, 2014, 34, 876.)

[4] Tang, M.; Xing, M.; Cai, M.-Q.; Hu, W.-H. Chin. J. Org. Chem. 2014, 34, 1268 (in Chinese). (唐敏, 邢栋, 蔡茂强, 胡文浩, 有机化学, 2014, 34, 1268.)

[5] Bae, I.; Han, H.; Chang, S. J. Am. Chem. Soc. 2005, 127, 2038.

[6] Cho, S. H; Yoo, E. J.; Bae, I.; Chang, S. J. Am. Chem. Soc. 2005, 127, 16046.

[7] Cassidy, M. P.; Raushel, J.; Fokin V. V. Angew. Chem., Int. Ed. 2006, 45, 3154.

[8] Cho, S. H.; Chang, S. Angew. Chem., Int. Ed. 2007, 46, 1897.

[9] Yoo, E. J.; Bae, I.; Cho, S. H.; Han, H.; Chang, S. Org. Lett. 2006, 8, 1347.

[10] Zhang, W.-S.; Kuang, C.-X.; Yang, Q. Chin. J. Chem. 2009, $27,1727$.
[11] Lu, P.; Wang, Y.-G. Chem. Soc. Rev. 2012, 41, 5687.

[12] Kim, S. H.; Park, S. H.; Choi, J. H.; Chang, S. Chem. Asian J. 2011, 6, 2618.

[13] Lu, P.; Wang, Y.-G. Synlett 2010, 165.

[14] Yoo, E. J.; Chang, S. Curr. Org. Chem. 2009, 13, 1766.

[15] Cheng, D.; Ling, F.; Li, Z.-X.;Yao, W.-J.; Ma, C. Org. Lett. 2012, 14, 3146.

[16] Yavari, I.; Nematpour, M.; Yavari, S.; SadeghizadehI, F. Tetrahedron Lett. 2012, 53, 1889.

[17] Xing, Y.-P.; Zhao, H.-Y.; Shang, Q.-Y.; Wang, J.; Lu, P.; Wang, Y.-G. Org. Lett. 2013, 15, 2668.

[18] Namitharana, K.; Pitchumani, K. Adv. Synth. Catal. 2013, $355,93$.

[19] Gouthaman, S.; Shanmugam, P.; Mandal, A. B. Tetrahedron Lett. 2013, 54, 3007.

[20] Shang, Y.-J.; Liao, K.-S.; He, X.-W.; Hu, J.-S. Tetrahedron 2013, 69, 10134.

[21] Yavari, I.; Nematpour, M.; Ghanbari, E. Mol. Diversity 2014, $18,721$.

[22] Periyaraja, S.; Shanmugam, P.; Mandal, A. B. Eur. J. Org. Chem. 2014, 2014, 954.

[23] Yao, B.-B.; Shen, C.; Liang, Z.-J.; Zhang, Y.-H. J. Org. Chem. 2014, 79, 936.

[24] Yavari, I.; Sheikhi, A.; Nematpour, M.; Taheri, Z. Synth. Commun. 2015, 45, 1089.

[25] Li, S.-Y.; Zou, S.-W.; Wu, J. Chem. Asian J. 2012, 7: 2882.

[26] Li, B.-S.; Yang, B.-M.; Wang, S.-H.; Zhang, Y.-Q.; Cao, X.-P.; Tu, Y.-Q. Chem. Sci. 2012, 3, 1975.

[27] Cheng, G.-L.; Cui, X.-L. Org. Lett. 2013, 15, 1480.

[28] Wang, J.-J.; Wang, J.; Lu, P.; Wang, Y.-G. J. Org. Chem. 2013, 78, 8816.

[29] Rajagopal, B.; Chen, Y.-Y.; Chen, C.-C.; Liu, X.-Y.; Wang, H.-R.; Lin, P.-C. J. Org. Chem. 2014, 79, 1254.

[30] Sun, L.; Zhu, Y.-X.; Lu, P.; Wang, Y.-G. Org. Lett. 2013, 15, 5894.

[31] Kumar, Y. K.; Kumar, G. R.; Reddy, M. S. J. Org. Chem. 2014, 79, 823.

[32] Zhang, D.; Nakamura, I.; Terada, M. Org. Lett. 2014, 16, 5184.

[33] Mahendran, V.; Shanmugam, S. RSC Adv. 2015, 5, 20003.

[34] Li, S.; Wu, J. Chem. Commun. 2012, 48, 8973.

[35] Shen, Y.; Han, C.; Cai, S.-Y.; Lu, -P.; Wang, Y.-G. Tetrahedron Lett. 2012, 53, 5671.

[36] Jiang, Z.; Lu, P.; Wang, Y.-G. Org. Lett. 2012, 14, 6266.

[37] Murugavel, G.; Punniyamurthy, T. Org. Lett. 2013, 15, 3828.

[38] Zhou, F.-G.; Liu, X.; Zhang, N.; Liang, Y.-J.; Zhang, R.; Xin, X.-Q.; Dong, D.-W. Org. Lett. 2013, 15, 5786.

[39] Jiang. B.; Tu, X.-J.; Wang, X.; Tu, S.-J.; Li, G. Org. Lett. 2014, 16, 3656.

[40] Cui, S.-L.; Lin, X.-F.; Wang, Y.-G. Org. Lett. 2006, 8, 4517.

[41] Mandal, P. K. RSC Adv. 2014, 4, 5803.

[42] Ramanathan, D.; Pitchumani, K. Eur. J. Org. Chem. 2015, 2015, 463.

(Lu, Y.) 